\title{
Relación entre orientación política y condición socioeconómica en la cultura política chilena: una aproximación desde la psicología política*
}

\author{
Andrés Haye \\ Héctor Carvacho \\ Roberto González \\ Jorge Manzi** \\ Carolina Segovia***
}

Resumen: A partir de un estudio longitudinal sobre cultura política en chilenos, discutimos la paradojal evidencia encontrada en la literatura que plantea que, por un lado, las actitudes políticas más pro-democráticas y anti-autoritarias se observarían en sectores sociales acomodados y, por otro lado, las actitudes más pro-conservadoras y anti-igualitaristas se observarían en grupos sociales dominantes. Nuestros datos muestran que el patrón de actitudes políticas (autoritarismo, apoyo a la democracia, conservadurismo y nacionalismo) es similar entre personas de derecha de estrato alto, izquierda de estrato bajo, y centro de estrato medio. En cambio, quienes manifiestan una orientación política incongruente con su condición socioeconómica presentan actitudes políticas diferenciadas del patrón predominante. Concluimos que la paradoja puede resolverse explicando el patrón de actitudes políticas de un grupo en función del (des)ajuste de la orientación política a sus condiciones socioeconómicas. tica, Chile.

Palabras clave: ideología, actitudes políticas, clase social, identidad polí-

\section{Relationship between political orientation and socioeconomic status in Chilean political culture: a political psychology approach}

\footnotetext{
Abstract: Based on a longitudinal study about political culture in Chile, we discuss the paradoxical evidence found in the literature suggesting, on the one

* La realización de este trabajo ha sido posible gracias al financiamiento otorgado al proyecto FONDECYT N 1050887 “Estudio Psicosocial de la Cultura Política de Tres Generaciones de Chilenos."

** Pontificia Universidad Católica de Chile, Santiago, Chile. Email: ahaye@uc.cl

*** Centro de Estudios Públicos, Santiago. Chile.
} 
hand, that the more pro-democratic and anti-authoritarian political attitudes are expected to be observed in higher economic level groups and, on the other hand, more conservative and anti-egalitarian attitudes are expected to be observed in dominant groups. We found that the pattern of political attitudes (authoritarianism, support to democracy, conservatism, and nationalism) is similar among rightwingers of high economic status, leftwingers of low economic status, and centrists of middle economic status. Instead, individuals whose political standing is incongruent with their socioeconomic group, show a pattern of political attitudes different from the predominant pattern. We conclude that the paradox can be solved explaining the pattern of political attitudes of a social group as a function of the fit of its political identity to its socioeconomic condition.

Key words: ideology, political attitudes, social class, political identity, Chili.

Recibido: 13.05.2009

Aceptado: 10.06.2009

\section{Introducción}

Desde que Marx comenzó a utilizar la noción de ideología en el sentido amplio que hasta hoy tiene, mucho se ha escrito sobre este tema en diversos campos de las ciencias sociales. En términos bien generales, esta noción se refiere al problema de las complejas, si no oscuras relaciones entre las ideas y creencias que sostiene una comunidad y sus condiciones sociales de vida. La sociología, la historia, la filosofía, la estética, la ciencia política, el psicoanálisis y la psicología social han abordado múltiples aspectos del problema de la ideología, sin que tales problemas lleguen a una resolución teórica clara, y han analizado distintos ámbitos del mundo contemporáneo utilizando la noción de ideología sin contar hasta hoy con un uso consensual preciso del concepto. Ello habla al mismo tiempo de lo elusivo y conflictivo que resulta este concepto, así como de su poder heurístico para estimular la reflexión y la investigación en estos diversos campos de las ciencias sociales.

En el uso que Marx le da al concepto de ideología, ésta se entiende, en el marco histórico de las sociedades modernas, como la producción social de la conciencia cuyo rendimiento psicológico permite que, bajo determinadas circunstancias, queden ocultas las fallas y contradicciones propias de mismo proceso social que la engendra, el capitalismo (Larraín 2007, Marx y Engels 1845/1974). Entre los rasgos centrales de la noción, cabe destacar por un lado que la ideología cumple la función de ocultamiento de la realidad social y, por otro, está anclada en los medios psicológicos de representación y de acción. Esta función de la llamada falsa conciencia se diferenciaría de las teorías del engaño del clero, que estaban presentes en el debate previo a Marx, pues en el caso de la ideología el ocultamiento no es consecuencia de un engaño deliberado sino de un sistema de producción de la conciencia funcional a la mantención y desarrollo del orden social vigente (Lenk 1974). La noción de un sistema de producción de la conciencia remite a la socialización de los individuos en determinados modos de per- 
cibir y ordenar el mundo, y en valores particulares mediante los cuales juzgar y jerarquizar la experiencia, así como actuar con sentido en el mundo. Este anclaje de la operación de ocultamiento en la configuración subjetiva de los individuos dependería de una organización social de prácticas de socialización que quedan institucionalizadas, por ejemplo, en las instancias de la familia y la escuela (Althusser 1997).

Ambas ideas, la función de ocultamiento y el anclaje psicológico de la ideología, han sido ampliamente discutidas en los últimos 150 años (ver por ejemplo Adorno y Horkheimer 1969, Althusser 1997, Jost 2006, Jost et al. 2009, Jost et al. 2008, Knight 2006, Larraín 2008, Lenk 1974); a lo largo de este artículo serán tematizadas específicamente a partir de la evidencia que hemos encontrado en un estudio sobre cultura política realizado en Santiago de Chile. Por un lado, la función de ocultamiento de la ideología será abordada en términos de la relación observada en el estudio entre las posiciones que toman las personas frente a diversos aspectos del mundo político y sus condiciones socioeconómicas de existencia. En este sentido, este artículo reporta los resultados del estudio con un foco específico en el cruce entre cultura política y nivel socioeconómico.

Por otro lado, el examen del anclaje psicológico de la ideología estará basado en la definición del objeto de estudio: las disposiciones psicológicas de los individuos. El enfoque metodológico de esta investigación implica un acercamiento a las formas de representación y del sentido de la acción específicamente en términos de actitudes políticas de las personas. La noción de actitud (ver Eagly y Chaiken 1993) integra las dimensiones cognitivas (representación) y motivacionales (acción) en la forma de tomas de posición evaluativas de los individuos frente a situaciones o elementos del mundo, en este caso, político (ver por ejemplo Robinson et al. 1999). Específicamente, la noción de actitudes políticas se refiere a tendencias psicológicas generalizadas de los individuos, es decir, a inclinaciones hacia una u otra toma de posición frente a aspectos estructurales o relativamente estables del mundo político (en contraste con aspectos puntuales o contingentes), como son la disposición favorable/desfavorable a relaciones autoritarias para mantener el orden (autoritarismo, ver Altemeyer 1998, Feldman 2003), la disposición positiva/negativa frente a instituciones del mundo político (como apoyo, confianza, etc., ver Citrin y Muste 1993, Segovia et al. 2008), o la disposición de promoción/resistencia al cambio social (conservadurismo, Jost et al. 2003a). Operacionalmente, las actitudes se infieren a partir de múltiples registros de tomas de posición con respecto a enunciados que afirman algo acerca de alguno de los aspectos mundo del político. Así, el presente artículo se inscribe en la tradición de la psicología social-política (por ejemplo, Adorno et al. 1950, Jost et al. 2009), específicamente en la perspectiva de entender la cultura política de un grupo como la articulación de actitudes políticas de los individuos que la componen (Almond y Verba 1965, Pye y Verba 1965, Jost et al 2009).

En psicología social, la definición más influyente de ideología es la presentada por Adorno y sus colaboradores (1950). Para ellos ideología es 
una organización de opiniones, actitudes y valores, una forma de pensar sobre el hombre y la sociedad. Varios de los más importantes autores en el área (Billig 1984, Jost 2006, Kerlinger 1984) han basado sus propios trabajos en esta definición. Nosotros igualmente adherimos a esta perspectiva, pero asumiremos las siguientes distinciones conceptuales para este artículo.

\section{Cultura política, ideología, actitudes políticas}

Llamaremos cultura política a la organización de creencias y valoraciones en relación al mundo político, sobre las que se sostienen las prácticas sociales desplegadas en aquel mundo político en una sociedad determinada, y que es diferente a la organización de creencias y valoraciones de otra sociedad en función de su historia social específica y de las características de su mundo político. Así, la cultura política de los chilenos será el modo en que predominantemente se organizan visiones y preferencias políticas entre los chilenos de manera relativamente estable en el tiempo. Esta definición teórica, por lo tanto, implica que operacionalmente es importante identificar creencias y valoraciones relativamente profundas o permanentes, en contraste con elementos contingentes. Este concepto de cultura política, por tanto, denota algo muy similar que el concepto antes citado de ideología: «organización de opiniones, actitudes y valores». Sin embargo, se trata de un nivel de descripción diferente. La noción de cultura política implica que es común a los miembros de un grupo. Sin embargo, típicamente el mundo político es objeto de controversias que se estructuran de manera sistemática en torno a orientaciones ideológicas particulares (Billig et al. 1988). El carácter ideológico de la cultura política radica justamente en la diferenciación de creencias y valoraciones dentro de la sociedad. Ciertamente en una cultura política típicamente hay unos pocos principios totalmente compartidos entre los miembros de una sociedad, pero tales principios adquieren un valor ideológico (y eventualmente de falsa conciencia) solamente desde el punto de vista de valores alternativos que pueden provenir de otras sociedades o épocas, así como imaginarse a partir de la negación o modificación reflexiva de los propios principios comúnmente presupuestos.

Aquí entendemos la ideología como una orientación de creencias y valoraciones específica dentro de una sociedad, constituida por su diferencia con otras orientaciones dentro de la misma sociedad. Por ejemplo, esta distinción será utilizada para identificar movimientos particulares de ciertos grupos al interior de la cultura política chilena que, diferenciándose como una mayoría o, alternativamente, diferenciándose de la mayoría, establecen modos particulares de manifestarse ideológicamente, siguiendo un patrón específico de configuración de actitudes políticas que será discutido hacia el final del artículo. La configuración ideológica de un grupo sería, entonces, el perfil común de actitudes políticas que muestran los miembros de tal grupo. En este sentido, una ideología es el patrón de actitudes común a un grupo desde el punto de vista de su diferencia políticamente relevante con otros patrones. Una cultura política se diferencia ideológica- 
mente en función de dimensiones (como por ejemplo autoritarismo, liberalismo, conservadurismo, nacionalismo, etc.) que son relevantes para la estructuración de las prácticas sociales ligadas a un mundo político determinado. Por ende, entre dos grupos ideológicos, por ejemplo uno pro democrático y otro anti democrático, la dimensión del apoyo a la democracia constituye un aspecto estructural común de la cultura política y al mismo tiempo un factor relevante de diferenciación ideológica.

Finalmente, a un nivel más concreto, haremos una distinción entre orientación política y configuración ideológica. La primera la entendemos como la posición de un sujeto en términos de las oposiciones como por ejemplo izquierda/derecha, liberal/conservador, democracia/autoritarismo, pro/anti gobierno, etc., o sea de polaridades simbólicas generalizadas. En este estudio hemos operacionalizado la orientación política de las personas como su autoposicionamiento en una dimensión izquierda-derecha, que es la más ampliamente usada en la investigación (Jost 2006) y la que resulta más familiar al lenguaje de la cultura política chilena (Colomer y Escatel 2005, Navia 2004, 2005, 2007, Ortega 2003). En este sentido, la orientación política puede esperarse que sirva como un resumen abstracto de la ideología, o configuración ideológica, en cuanto manera particular de organización de múltiples actitudes políticas, como son las disposiciones autoritarias, el nivel de apoyo a la democracia, el grado de resistencia al cambio social, la confianza hacia instituciones políticas particulares, etc., que en su conjunto comportan una complejidad y multiplicidad de niveles que no tiene la orientación política (Jost et al. 2009). Si la orientación política es o no un buen resumen de la ideología de un grupo en una cultura política determinada, es una cuestión empírica que se ha abordado en diversos contextos y con múltiples medios (Colomer y Escatel 2005, Jost 2006, Jost et al. 2009, Knight 1999). ${ }^{1}$

\section{Ideología y condición socioeconómica}

La relación entre ideología y nivel de acceso económico, así como la posición en la estructura económica, ha sido planteada muy tempranamente en la discusión en ciencias sociales. En la tradición marxista, esta relación cobra relevancia en las obras de Lukács y Althusser. El primero,

\footnotetext{
${ }^{1}$ La investigación que está a la base del presente artículo incluyó un extenso análisis de este asunto, cuyos resultados muestran que el eje izquierda-derecha es un indicador válido e informativo de diferencias ideológicas relevantes en la cultura política chilena actual: es una medida que muestra alta estabilidad a través del tiempo, que permite predecir la opinión de los chilenos en tópicos controversiales de discusión pública y resumir tanto las diferencias entre los partidos políticos, como la identificación con estos; además, las diferencias en las actitudes políticas entre quienes se clasifican en los polos de izquierda y derecha son consistentes con lo mostrado en la literatura, siendo entre otros el autoritarismo, el conservadurismo y el apoyo a la democracia aspectos que diferencian claramente a la izquierda de la derecha (Brunner 1990). Por razones de espacio, estos resultados no se reportan en este artículo sino en otro trabajo en preparación.
} 
influido por la obra de Lenin, plantea un concepto neutro de ideología, diferenciándose de los planteamientos de Marx (Larraín, 2008) que no implican que la consciencia sea siempre y necesariamente falsa o encubridora. Para Lúkacs, ideología se identifica con el concepto de conciencia de clase, que puede ser psicológica, cuando corresponde a ideas psicológicamente explicables que las personas tienen sobre su situación de vida; o adscrita, cuando corresponde a reacciones propias a una posición particular en el proceso de producción (Lukács, 1969). Althusser (1997) propone que la ideología aparece como cierta representación del mundo que liga a los hombres con sus condiciones de existencia y a los hombres entre sí; la ideología está presente en todas las actividades del hombre y sólo sería inteligible a través de su estructura, pues se diferenciaría en las diferentes formaciones sociales existentes, por ejemplo las clases sociales.

En psicología social Adorno et al. (1950) propusieron que la personalidad autoritaria era más común en la clase trabajadora. Esta relación ha sido largamente estudiada, habiendo cierto consenso en que las personas de menores ingresos y menor nivel educacional tienen mayores niveles de autoritarismo (Napier y Jost 2008, Schuman et al. 1992). Esta situación se ve reflejada en un mayor apoyo a las autoridades (Carlin 2006). Sin embargo, no hay consenso en las razones que explican esta situación. El influyente trabajo de Lipset (Lipset 1960, Lipset y Raab 1978) sobre el "autoritarismo de clase obrera” sugiere que la intolerancia se concentra especialmente en los grupos económicamente desaventajados. Este trabajo es revisado en detalle por Napier y Jost (2008) a la luz de la teoría de justificación del sistema. La teoría de la justificación del sistema busca superar lo propuesto previamente por las teorías de la identidad grupal (Tajfel y Turner 1986) y de la dominancia social (Pratto, Sidanius y Levin 2006, Sidanius 1993, Sidanius y Pratto 1999). Según la teoría de la justificación del sistema, las personas no solo tienen actitudes favorables hacia su propio grupo, sino que también hacia el sistema social existente y el statu quo (Jost y Hunyady, 2002; 2005; Jost, Banaji, y Nosek, 2004). Esto sucedería, al menos en parte, porque existe una razón ideológica para justificar el orden social existente. Esta razón ideológica conduciría a desplegar favoritismo por el exogrupo, por sobre el grupo propio, y a la internalización de la inferioridad de los grupos desaventajados, que se manifestaría principalmente en un nivel no consciente o implícito; y que esto es más fuerte en los grupos desaventajados en el orden social (op cit). Como hipótesis plantean que una explicación para esto puede ser el desarrollo de las capacidades adaptativas de acomodarse, internalizar y racionalizar las claves ambientales, especialmente las que dificultan o imposibilitan el cambio (Jost, Banaji y Nosek 2004).

Adicionalmente, el mismo Jost ha encontrado una correlación positiva entre la orientación política de derecha y los ingresos (Carney et al. 2008), y que el ingreso es un predictor de derechismo, aunque más débil que la religiosidad, autoritarismo (convencionalismo y absolutismo moral), la intolerancia y el conservadurismo económico (Napier y Jost 2008). Estos hallazgos son inconsistentes con la evidencia indicada anteriormente. 
En otras palabras, la investigación nos ofrece un cuadro ambiguo. Por un lado, las actitudes políticas más pro-democráticas y más anti-autoritarias se observarían en sectores sociales más acomodados, pues son el producto de un proceso de educación más sofisticado, que permite a las personas desarrollar actitudes más elaboradas (Adorno et al. 1950, Jost et al. 2003, Kemmelmeier 2008, Michaud et al. 2009). Esta tesis, si embargo, no toma suficientemente en cuenta las diferencias ideológicas al interior de un mismo sector socioeconómico. Por otro lado, las actitudes más proconservadoras y más anti-igualitaristas se observarían en grupos sociales dominantes, interesados en la mantención de jerarquías de poder y de acceso económico (ver por ejemplo Sidanius y Pratto 1999), mientras que lo contrario se daría en grupos oprimidos o de bajo status social, más favorables a la promoción del cambio social en pos de la igualdad. Esta segunda tesis, cuya predicción es totalmente contraria a la primera, tampoco toma en cuenta las diferencias ideológicas al interior de un mismo sector socioeconómico. En ambos casos se establece una relación simple entre posición ideológica y posición socioeconómica. El presente artículo aborda este problema de la relación entre la posición ideológica y la posición económica de las personas, con la hipótesis que ésta es una relación compleja, que no permite explicar la configuración ideológica de las personas como función lineal de su condición socioeconómica. En particular, en el artículo abogamos, con evidencia empírica, por un modelo que se centra en las diferencias ideológicas al interior de un mismo sector socioeconómico y que permite conjugar de manera coherente las dos tesis presentes en la literatura, a pesar que parezcan contradictorias. En concreto, en este estudio hemos analizado la configuración ideológica de las personas en términos de aquellas actitudes políticas que han estado especialmente vinculadas a esta discusión sobre posición ideológica y posición económica, a saber: autoritarismo, apoyo a sistemas democráticos, conservadurismo y nacionalismo.

\section{El estudio Diseño y muestra}

Para indagar en la cultura política de los chilenos hemos realizado un estudio longitudinal con tres mediciones a los mismos sujetos, separadas por intervalos de aproximadamente 14 meses. La muestra se estratificó por grupo de edad y nivel de acceso económico. Distinguimos tres niveles socioeconómicos caracterizados por sus ingresos y su estándar de vida, que se podrían clasificar en:

- Alto: ingresos familiares mayores a \$2.000.000 (U\$3,250) mensuales aproximadamente, correspondiente al $10 \%$ superior de la población (Ministerio de Planificación, Gobierno de Chile 2006).

- Medio: ingresos familiares entre $\$ 450.000$ (U\$750) y $\$ 2.000 .000$ (U\$3,250) mensuales aproximadamente, correspondiente al $50 \%$ de la población (op. cit.). 
- Bajo: ingresos familiares menores a \$450.000 (U\$750) mensuales aproximadamente, correspondiente al 40\% de la población (op. cit.).

El diseño incluyó diferentes grupos etarios porque se ha evidenciado diferencias ideológicas asociadas a cohortes de edad (Alwin y Krosnick 1991). Existe una perspectiva bien asentada sobre la estrecha relación entre la pertenencia a un grupo etáreo socializado bajo condiciones específicas y su configuración ideológica (Duncan y Agronick 1995, Schuman y Scott 1989, Sears 1990, Steward y Healy 1989). Complementariamente, en el campo de la socialización política, así como en investigaciones desarrolladas a partir de la teoría del ciclo de vida, se coincide en definir a la etapa de la adolescencia tardía o de la adultez temprana como el período más decisivo para perfilar opiniones, actitudes y orientaciones en torno a lo político (Sears y Levy 2003, Steward y Healy 1989). Así por ejemplo, hay un alto consenso en la asociación entre volverse mayor y adoptar actitudes políticas más conservadoras (Glenn 1974, Ross 1989), situación que ya se ha observado en Chile (Luna 2008).

Para distinguir los grupos de edad nos basamos en la hipótesis de que los contextos de socialización política podrían configurar diferencias ideológicas relevantes entre los grupos (por ejemplo en Mcdevitt y Chaffee 2002, ver también Dawson y Prewitt 1969, Jennings y Niemi 1981). Definimos tres grupos etáreos:

- Mayores: 53 - 63 años, que cumplieron 18 años antes del golpe militar de 1973, habiéndose socializado políticamente durante los gobiernos democráticos previos al golpe (presidencias de Frei Montalva y Allende).

- Adultos: 36 - 43 años, que cumplieron 18 antes de volver a la democracia y que habrían participado activamente en el plebiscito que condujo al retorno a la democracia (Toro 2008).

- Jóvenes: 20 - 27 años, que cumplieron 18 en torno a la elección presidencial del 2005 de Michelle Bachelet y cuya socialización política ha trascurrido principalmente dentro de un régimen democrático post-dictatorial.

Diseñamos un muestreo mixto, en el que seleccionamos de forma aleatoria un conjunto de manzanas del Gran Santiago segmentadas por nivel socioeconómico. ${ }^{2}$ A cada manzana le asignamos de forma aleatoria hasta 5 sujetos de acuerdo a cuotas por sexo y edad. Sobrerepresentamos al grupo socioeconómico alto intencionalmente para garantizar tamaños muestrales equivalentes para cada grupo, para permitir comparabilidad intergrupal. El contacto se realizó en los domicilios de los sujetos, por

\footnotetext{
${ }^{2}$ Si bien la muestra está limitada al Gran Santiago, en el presente artículo aludimos a la cultura política "chilena” actual. Reconocemos que este es un giro inadecuado, pues implicaría una generalización indebida si se usara en sentido estricto. Nosotros usamos la expresión aquí en sentido lato.
} 
encuestadores capacitados específicamente para tales efectos.

En la primera medición, realizada el segundo semestre de 2005, encuestamos a 996 casos válidos, siendo el $27 \%$ de los casos de nivel socioeconómico alto, 37\% medio, y 36\% bajo. El 52\% de los casos fueron mujeres y el $48 \%$ hombres. El 33\% correspondió al grupo etario mayor, el $33 \%$ al grupo adulto y un 34\% al joven. Esta medición fue hecha 4 meses antes de la primera ronda de la elección presidencial del 2005.

La segunda medición fue realizada el segundo semestre del 2006. En ella recontactamos a 695 participantes encuestados el año anterior. Adicionalmente, contactamos a 146 sujetos para reemplazar la pérdida muestral. La composición muestral es equivalente en términos de niveles socioeconómico, sexo y edad de los participantes.

La tercera medición se realizó el primer semestre de 2008 y se recontactó a 663 sujetos. De estos, 494 fueron encuestados en las tres mediciones, 73 en la primera y la tercera medición, y 96 sujetos en la segunda y la tercera medición. La composición muestral también se mantuvo para esta medición.

La pérdida muestral para los sujetos que participaron en todas las mediciones es de 50,4\% entre el año 1 y el 3 . Los sujetos que participaron en al menos dos mediciones corresponden al 37,2\%, mientras que en solo una medición participó el 13,2\% de los sujetos.

En la introducción se planteó que la cultura política es una organización relativamente estable de actitudes respecto del mundo político en una sociedad determinada. Es importante considerar la relativa estabilidad de la relación entre tales elementos a través de una ventana temporal, pues de otra manera no puede distinguirse empíricamente la cultura política propiamente tal de aquellas actitudes y opiniones contingentes, que son más superficiales desde el punto de vista de la noción de ideología y son, por lo mismo, más variables en el tiempo. Esta ha sido, en efecto, la principal razón del diseño longitudinal del presente estudio, en que hemos cubierto una ventana temporal de tres años y medio. Así, para caracterizar la cultura política de los chilenos identificaremos el patrón de interrelación de algunos de estos elementos actitudinales predominantemente a través de las tres mediciones.

\section{Medidas de posicionamiento ideológico y actitudes políticas}

\section{Orientación política}

Para estimar la orientación política de los participantes les hemos pedido en cada año de medición que se posicionen en una escala de 1 a 9, donde 1 es izquierda, 5 es centro y 9 es derecha. Jost (2006) y Knight 
(1999) han mostrado que ésta es una forma válida y confiable de medir la identificación con las orientaciones políticas de izquierda y derecha.

Los resultados arrojan un promedio de 5,16 en la primera medición, 5,07 en la segunda, y 5,14 en la tercera, sin que existan diferencias estadísticamente significativas entre estas mediciones $(F=2,768$; $p<0,05)$, lo que muestra la estabilidad en el tiempo del autoposicionamiento. Esta estabilidad, en conjunto con la alta correlación que hay entre las tres mediciones de la orientación política (ver Tabla 1), atestiguan acerca de la confiabilidad de esta medida.

Tabla 1: Correlaciones entre las mediciones de Orientación Política

\begin{tabular}{|rr|r|r|r|r|r|r|}
\hline & \multicolumn{2}{|l|}{ Medición 1 } & \multicolumn{2}{l|}{ Medición 2 } & \multicolumn{2}{l|}{ Medición 3 } \\
\hline Medición 1 & Correlación de Pearson & 1 & & 0,654 & $* * *$ & 0,659 & $* * *$ \\
\hline $\mathrm{N}$ & 922 & & 603 & & 477 & \\
\hline Medición 2 & Correlación de Pearson & & & 1 & 0,629 & $* * *$ \\
\hline $\mathrm{N}$ & & & 761 & & 473 & \\
\hline Medición 3 & Correlación de Pearson & & & & & 1 & \\
\hline $\mathrm{N}$ & & & & & 585 & \\
\hline
\end{tabular}

$* * * \mathrm{p}<0,001$

\section{Autoritarismo}

Desde la publicación de La Personalidad Autoritaria (Adorno et al. 1950), el autoritarismo ha estado en el centro de la investigación en psicología política. En esta obra, se plantea que existe una cierta configuración de personalidad que se asocia con las manifestaciones políticas de intolerancia extrema y particularmente con el fascismo. Ésta sería la personalidad autoritaria, y estaría altamente relacionada con el derechismo y el conservadurismo (Adorno et al. 1950). Los planteamientos de Adorno han sido ampliamente discutidos, por ejemplo en Billig $(1984,1986)$, siendo hasta hoy objeto de controversias. ${ }^{3}$

En nuestro estudio entendemos el autoritarismo como la creencia en que el orden social sólo es posible a través del control represivo de los ciudadanos por parte de la autoridad política que se percibe como legítima, y consecuentemente a través de la sumisión a tal autoridad por parte de los ciudadanos.

\footnotetext{
${ }^{3}$ Una revisión detallada de la relación entre autoritarismo y orientación política se puede encontrar en Meloen (1993). La relación entre autoritarismo y conservadurismo político se revisa en detalle en Stenner (2005).
} 
El concepto, por tanto, contiene dos aspectos. Por un lado, la creencia en que una autoridad política fuerte debe controlar que la vida social se mantenga en orden a través de la represión (prohibición, criminalización, discriminación, etc.) de conductas que impliquen desobediencia o no conformidad a la autoridad o a las normas establecidas, si es necesario por medio de la violencia abierta. Este aspecto del autoritarismo político se contrapone a los valores democráticos de igualdad de derechos y de tolerancia política. Por otro lado, la creencia en que la actitud cívica correspondiente es la obediencia incondicional a la autoridad, en oposición a la mayor autonomía personal. Las escalas más populares distinguen 3 componentes: la sumisión, la agresión autoritaria, y el convencionalismo (Altmeyer 1981, 1998, Funke 2005). Nosotros consideramos sólo los 2 primeros (que han mostrado mayor correlación y valor predictivo), pues el convencionalismo se incluye también en el conservadurismo, que discutiremos más adelante.

La medición de autoritarismo fue hecha con una escala compuesta por 5 ítems, principalmente del componente de agresión autoritaria, con los que se debía manifestar el grado de acuerdo o desacuerdo en un formato de 1 a 5 puntos. Presentó una alta y estable confiabilidad $\left(\alpha_{1}=0,71 ; \alpha_{2}=\right.$ 0,$\left.76 ; \alpha_{3}=0,76\right)$. Las preguntas son:

- Más que partidos y programas políticos, lo que nos hace falta es un líder que resuelva los problemas (Latinobarómetro).

- Los gobiernos deben ocupar mano dura cada vez que hay dificultades (Latinobarómetro).

- En vez de tanta preocupación por los derechos de las personas, lo que este país necesita es un gobierno firme (RWA, Altmeyer, 1998).

- En este país las sentencias que reciben los delincuentes son demasiado blandas (Radicalism-conservatism Scale, Comrey y Newmeyer, 1965)

- Las verdaderas claves para una sociedad exitosa son la obediencia y la disciplina (RWA, Altmeyer 1998).

\section{Apoyo a la democracia}

Tironi y Agüero (1999), Mainwaring y Torcal (2003) y Luna (2008) han identificado como el principal elemento de diferenciación política en Chile el eje autoritarismo-democracia, o la adhesión o rechazo a la dictadura. Esta situación se reforzaría por el sistema de elección parlamentaria que incentiva la existencia de dos bloques, los que apoyaron la dictadura militar de Pinochet (derecha) y los que se opusieron (izquierda). Según Toro (2008), comparando los niveles de aceptación a la democracia al interior del continente, Chile estaría entre los países con más bajo nivel de apoyo. 
El apoyo a la democracia fue medido con una escala de 3 ítems, que descompone una pregunta de tres alternativas usada en el Latinobarómetro en tres preguntas de acuerdo o desacuerdo de 1 a 5 puntos. La escala presentó una confiabilidad media y estable $\left(\alpha_{1}=0,58 ; \alpha_{2}=0,62 ; \alpha_{3}=0,57\right)$. Las preguntas usadas fueron:

- La democracia es preferible a cualquier otra forma de gobierno.

- En algunas circunstancias, un gobierno autoritario puede ser preferible a uno democrático. (Invertido)

- A la gente como yo, nos da lo mismo un régimen democrático que uno no democrático. (Invertido)

\section{Conservadurismo}

A partir de lo expuesto por Jost et al. (2003a), el conservadurismo comprende dos aspectos: la resistencia al cambio y la aceptación de diferencias sociales. Sin embargo, en su propia revisión el conservadurismo es tratado como una ideología, visión o paradigma, en el cual estos dos aspectos aparecen articulados de manera significativa en términos de una serie de actitudes y creencias, entre las cuales se distinguen seis: (A) convencionalismo: alta conformidad conductual y actitudinal a las convenciones (normas sociales) que son percibidas como apoyadas por la sociedad y sus autoridades (Wilson, 1973); (B) tradicionalismo: alta valoración de las tradiciones que constituyen la herencia histórica de la sociedad, particularmente de tradiciones religiosas (Wilson, 1973); (C) temor al desorden: extrema valoración de mecanismos que aseguren el orden cívico y la tranquilidad en la vida social (Wilson, 1973); (D) naturalismo: creencia que la sociedad tiene bases naturales que deben reconocerse y respetarse, incluyendo eventualmente la creencia que ciertas diferencias sociales tienen un sustrato natural (Muller, 2001); (E) justificación del sistema: creencia que la supervivencia histórica de las instituciones o normas sociales se debe a que satisfacen alguna necesidad o función (Muller, 2001); (F) foco en la prevención: preferencia por la evitación de cambios riesgosos por sobre la promoción de cambios radicales (Higgins, 1998).

En este estudio fueron medidos dos de los componentes del conservadurismo: el tradicionalismo y el temor al desorden. El tradicionalismo fue medido con una escala de 7 preguntas de 5 puntos desde muy en desacuerdo a muy de acuerdo. La confiabilidad de la escala fue alta y estable $\left(\alpha_{1}=0,63 ; \alpha_{2}=0,72 ; \alpha_{3}=0,72\right)$. Los ítems usados fueron:

- Este país tendría muchos menos problemas si se fortaleciera más la familia. (NES) dad. (NES)

- Las nuevas ideas y estilos de vida están debilitando nuestra socie- 
- Nuestro país estaría mucho mejor si se le diera más importancia a la religión. (Radicalism-conservatism Scale, Comrey y Newmeyer, 1965)

- Este país tiene problemas porque todos los días están tratando de cambiar algo. (Sólo años 2 y 3)

- Este país estaría mejor si se valorara cómo se hacían las cosas en el pasado. (Sólo años 2 y 3) años 2 y 3)

- Cuando las cosas están bien, no hay para qué cambiarlas. (Sólo

- Quien vive en este país debe respetar las normas de la sociedad chilena. (Stellmacher y Petzel 2005)

El temor al desorden fue medido con una escala de 5 preguntas de 5 puntos desde muy en desacuerdo a muy de acuerdo. Su confiabilidad fue de $\alpha_{1}=0,61 ; \alpha_{2}=0,75 ; \mathrm{y}_{3}=0,75$. Los ítems usados fueron:

- Las autoridades deberían preocuparse de hacer de Chile un país seguro. país.

- La seguridad ciudadana es lo más importante para el progreso del

- La única forma de que las personas no se metan en problemas es que respeten las normas de la sociedad. (Sólo años 2 y 3)

- Mientras más libertad se le da a la gente, más desorden hay en la sociedad. (Sólo años 2 y 3)

- Las leyes dan demasiada protección a los criminales. (Radicalismconservatism Scale, Comrey y Newmeyer, 1965) (Sólo años 2 y 3)

\section{Nacionalismo}

Se define como la aceptación irreflexiva de las autoridades nacionales, estatales y políticas, combinada con la creencia en la superioridad de la propia nación (Adorno et al. 1950, Skitk 2005). Se espera que en las personas de derecha y las personas de nivel socioeconómico bajo hayan niveles más altos de nacionalismo (Adorno et al. 1950, Billig 1995, Billig y Núñez 1998, Skitk 2005). Esta variable fue medida a través de una escala de 3 preguntas que presenta alta confiabilidad $\left(\alpha_{1}=0,73 ; \alpha_{2}=0,73 ; \alpha_{3}=0,77\right)$. Las preguntas, que debían ser respondidas en un formato de 5 puntos desde muy en desacuerdo a muy de acuerdo, fueron: rica Latina.

- Chile debiera ser un país económicamente dominante en Amé-

- Los países vecinos estarían mucho mejor si Chile influyera más en ellos. 
- Me importa mucho que Chile sea el número uno en todo lo que hace.

Estas cinco medidas de actitudes políticas se han construido como escalas, es decir como constructos basados en una multiplicidad de indicadores observables, que a su vez son el posicionamiento de cada persona frente los itemes que conforman la escala. Ello le otorga a las medidas mayor estabilidad. Los puntajes de los itemes fueron promediados para dar cuenta del puntaje de la escala. Por ende, el rango de valores posibles de las cinco escalas es de 1 a 5 . Los itemes fueron codificados de modo que mayores puntajes siempre significan mayores niveles del atributo.

\section{Resultados \\ Caracterización general de la cultura política chilena}

En primer lugar se presentan los promedios en la orientación política de los grupos socioeconómicos y etarios (ver Tabla 2). Para evaluar las diferencias de promedios, hemos realizado un análisis de varianza factorial con los valores del autoposicionamiento izquierda-derecha como variable dependiente. Por un lado, puede notarse que los niveles socioeconómicos altos tienen un promedio mayor que los otros dos en todos los años. Esta diferencia, estadísticamente significativa ( $\mathrm{p}<0,001)$, muestra que las personas de nivel socioeconómico alto tienen una orientación política más de derecha que las de otros grupos socioeconómicos. Por otro lado, entre los grupos de edad no se presentan diferencias estadísticamente significativas ( $p<0,01$ ) en la orientación política de las personas. Ambas observaciones son constantes a través de las tres mediciones.

\section{Tabla 2: Promedio en orientación política por nivel socioeconómico y grupo etario}

\begin{tabular}{|r|r|r|r|r|l|l|l|l|l|}
\hline & Med. 1 & N & DS & Med. 2 & N & DS & Med. 3 & N & DS \\
\hline $\begin{array}{r}\text { Nivel } \\
\text { socioeconómico }\end{array}$ & & & & & & & & & \\
\hline Bajo & 4,901 & 314 & 2,15 & 4,90 & 263 & 1,784 & 4,847 & 203 & 1,778 \\
\hline Medio & 4,853 & 339 & 1,956 & 4,882 & 321 & 1,769 & 4,799 & 209 & 1,786 \\
\hline Alto & 5,892 & 260 & 1,890 & 5,684 & 177 & 1,672 & 5,896 & 173 & 1,639 \\
\hline Grupo etario & & & & & & & & & \\
\hline Jóvenes & 5,164 & 323 & 1,955 & 4,985 & 273 & 1,667 & 5,014 & 213 & 1,755 \\
\hline Adultos & 4,933 & 297 & 2,136 & 5,182 & 225 & 1,827 & 5,269 & 182 & 1,859 \\
\hline Mayores & 5,377 & 300 & 2,073 & 5,073 & 262 & 1,861 & 5,138 & 189 & 1,793 \\
\hline
\end{tabular}

Nota: Todas las comparaciones según nivel socioeconómico resultaron significativas ( $<<0,001)$. Según grupo etario, en cambio, sólo hay diferencias estadísticamente significativas $(\mathrm{p}<0,05)$ en la primera medición, entre adultos y mayores. 
La Tabla 3 muestra el promedio de la muestra en cada una de las actitudes políticas incluidas en el estudio. A partir de estos promedios puede afirmarse que en la cultura política chilena existen niveles medianamente altos (considerando que el punto medio de la escala es 3) de autoritarismo, apoyo a la democracia, tradicionalismo y nacionalismo; y valores altos de temor al desorden.

Para evaluar la estabilidad en el tiempo de estas actitudes, se realizó para cada una de ellas un análisis de varianza de mediciones repetidas. Como puede apreciarse por medio de los índices de significación en la misma Tabla 3existen diferencias estadísticamente significativas entre las mediciones de autoritarismo, tradicionalismo y nacionalismo; no así en apoyo a la democracia. Todas estas diferencias consisten en un aumento a lo largo del tiempo, lo que indica una cierta “derechización” de la muestra, pero que no se ve reflejada en la medición de orientación política, tal como queda sugerido por la ausencia de cambio significativo a través de las tres mediciones de esta variable.

Tabla 3: Promedio en actitudes políticas en cada medición

\begin{tabular}{|l|c|c|c|}
\hline & Medición 1 & Medición 2 & Medición 3 \\
\hline Autoritarismo*** & 3,58 & 3,72 & 3,85 \\
\hline Apoyo a la democracia & 3,69 & 3,68 & 3,74 \\
\hline Tradicionalismo*** & 3,50 & 3,54 & 3,69 \\
\hline Temor al desorden & 4,12 & 3,98 & 4,18 \\
\hline Nacionalismo*** & 3,70 & 3,79 & 3,85 \\
\hline
\end{tabular}

$* * * \mathrm{p}<0,001 ; * \mathrm{p}<0,05$

Al comparar estas actitudes entre los grupos de edad se encuentran diferencias estadísticamente significativas en las variables relacionadas al conservadurismo (tradicionalismo y temor al desorden), tal cual se esperaba según la literatura y otras mediciones en Chile. Además hay diferencias en el nacionalismo. Todas estas diferencias indican que los grupos mayores son más “derechizados” que los más jóvenes. Sin embargo, a pesar de las diferencias, el patrón de los puntajes al interior de estos grupos etarios es similar al de toda la muestra (Tabla 4, página siguiente).

Por último, al comparar por nivel socioeconómico se obtienen diferencias estadísticamente significativas $(p<0,001)$ en todas las variables, como se muestra en la Tabla 5. Concordantemente con lo sugerido por la literatura, las personas de nivel socioeconómico bajo tienen mayores montos de autoritarismo, tradicionalismo, nacionalismo y temor al desorden. 
Tabla 4: Promedio en actitudes políticas por grupo etario

\begin{tabular}{|l|c|c|c|}
\hline & Jóvenes & Adultos & Mayores \\
\hline Autoritarismo & 3,588 & 3,695 & 3,709 \\
\hline Apoyo a la democracia & 3,753 & 3,709 & 3,777 \\
\hline Tradicionalismo*** & 3,279 & 3,602 & 3,742 \\
\hline Temor al desorden*** & 3,953 & 4,044 & 4,173 \\
\hline Nacionalismo** & 3,610 & 3,842 & 3,808 \\
\hline
\end{tabular}

$* * * \mathrm{p}<0,001 ; * * \mathrm{p}<0,01$

Por el contrario, el apoyo a la democracia aumenta en los niveles socioeconómicos más altos. Igual que en el caso de las diferencias encontradas según grupo etario, las diferencias según nivel socioeconómico tampoco afectan las tendencias globales respecto a los puntajes en este conjunto de actitudes. Sin embargo, a diferencia de lo que ocurre con el factor etario, que genera diferencias en algunas de las actitudes y con tamaños de efectos pequeños, el factor socioeconómico produce diferencias en todas las actitudes políticas y con tamaños de efecto de moderados a grandes.

Tabla 5: Promedio en actitudes políticas por nivel socioeconómico

\begin{tabular}{|l|c|c|c|}
\hline & Bajo & Medio & Alto \\
\hline Autoritarismo*** & 3,910 & 3,637 & 3,446 \\
\hline Apoyo a la democracia*** & 3,494 & 3,792 & 3,954 \\
\hline Tradicionalismo*** & 3,734 & 3,503 & 3,385 \\
\hline Temor al desorden*** & 4,224 & 4,025 & 3,921 \\
\hline Nacionalismo*** & 3,927 & 3,714 & 3,620 \\
\hline
\end{tabular}

$* * * \mathrm{p}<0,001$

En síntesis, la cultura política chilena actual se caracteriza por un núcleo estándar extensamente compartido, incluso intergeneracionalmente, en que coexisten un alto autoritarismo, apoyo a la democracia, tradicionalismo y nacionalismo; y valores muy altos de temor al desorden. Las principales diferencias son atribuibles a los niveles socioeconómicos, tanto en la orientación política como en el conjunto de actitudes políticas. Sin embargo, estas diferencias son paradojales, pues mientras en la orientación política el nivel socioeconómico alto es más de "derecha”, a nivel de las actitudes políticas el grupo socioeconómico bajo resulta más de "derecha”. Esta inconsistencia nos obliga a realizar una observación más detallada de la relación entre estas variables. 


\section{Relación entre orientación política y nivel socioeconómico en la cultura política chilena}

Para explorar la relación entre nivel socioeconómico y orientación política, hemos estimado los promedios de cada una de las actitudes políticas en los nueve grupos determinados por el cruce entre estos factores. Para ello hemos identificando como de izquierda a aquellos participantes que se autoposicionaron entre 1 y 3 , como de centro entre 4 y 6 , y como de derecha entre 7 y 9. La Figura 1 muestra los patrones de promedios para cada una de las cinco actitudes políticas estudiadas. En primer lugar, destaca cierta regularidad a través de las diversas actitudes: además del efecto del nivel socioeconómico ya descrito (Tabla 5), hay un efecto de la orientación política que consiste en observarse mayores montos de dichas actitudes en los grupos más de derecha. El patrón es equivalente pero inverso en relación con el apoyo a la democracia. En segundo lugar, en todas las actitudes se observa que los promedios extremos corresponden a los grupos de derecha de nivel socioeconómico bajo y de izquierda de estrato alto. Por ejemplo, el grupo que muestra mayor autoritarismo es el de derecha de nivel bajo, mientras que el menor autoritarismo está en la izquierda de nivel alto. Estos mismos grupos son los que presentan los promedios extremos de apoyo a la democracia, solo que en dirección opuesta. Este patrón sistemático, que se observa al considerar el cruce entre nivel socioeconómico y orientación política, sugiere que es importante analizar el efecto conjunto de tales factores para entender las diferencias en los promedios de las actitudes políticas.

\section{Figura 1: Medias marginales estimadas para actitudes políticas según orientación política y nivel socioeconómico}

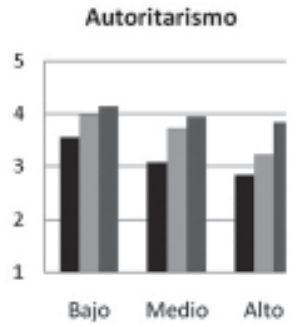

Nacionalismo

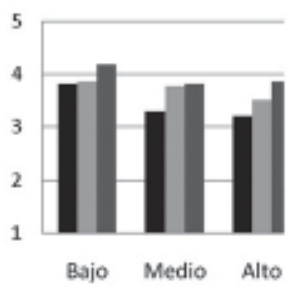

Apoyo a la democracia

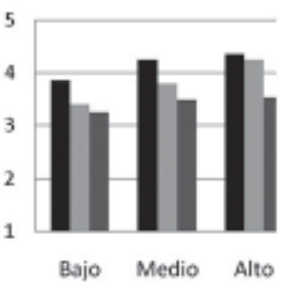

Temor al desorden

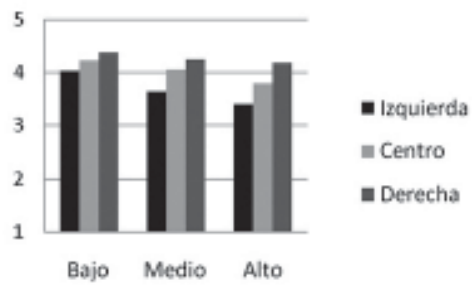

Tradicionalismo

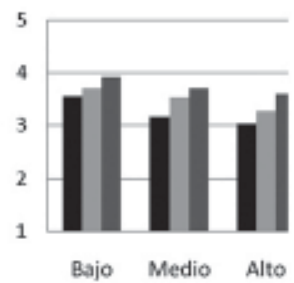


Al tomar en cuenta conjuntamente nivel socioeconómico y orientación política, se observa por ejemplo que los grupos izquierda/bajo, derecha/alto y centro/medio son similares en sus montos de tradicionalismo, temor al desorden y nacionalismo. Esto llama la atención pues resulta inesperado según las tesis presentes en la literatura acerca de la relación entre posición ideológica y posición socioeconómica. Observaciones de este tipo permiten hipotetizar que una clave para entender estos patrones de actitudes políticas radica en analizarlas en función de las diversas combinaciones de valores de orientación política con niveles de acceso económico. En otras palabras, planteamos la hipótesis de una interacción entre estos factores, en el sentido que la relación entre orientación política y actitudes políticas no es homogénea a través de distintos niveles socioeconómicos. Específicamente, probaremos que las combinaciones izquierda/bajo, derecha/alto y centro/medio entre posición ideológica y posición socioeconómica ofrecen un patrón de actitudes políticas similar entre sí y diferente de los que muestran las combinaciones izquierda/alto y derecha/bajo. Debe notarse que esta hipótesis no se refiere a una interacción estándar ${ }^{4}$ sino a una interacción especial que dice relación con el grado de congruencia entre la posición ideológica y la posición socioeconómica, en términos de lo que teóricamente se esperaría según buena parte de la literatura (Levin et al. 1998, Lukács 1969, Sidaius y Pratto 1999; cf. Adorno et al. 1950 y Jost et al. 2004). Por ejemplo, a la luz de la noción de conciencia de clase, las actitudes políticas más favorables a mantener el statu quo (derecha) se observarían en sectores dominantes y las actitudes más proclives al cambio social (izquierda) se observarían en grupos dominados. Así, a las combinaciones izquierda/bajo, derecha/alto y centro/medio las denominaremos operacionalmente congruentes, mientras que a las combinaciones izquierda/alto y derecha/bajo las llamaremos incongruentes. Aquellos grupos que manifiestan una orientación política que favorecería a los intereses esperados para su grupo socioeconómico, son los que llamamos congruentes, mientras que aquellos grupos que manifiestan una orientación política que atentaría contra los intereses esperados para su grupo socioeconómico son los que llamamos incongruentes.

La Tabla 6 muestra la cantidad de sujetos para cada uno de los nueve grupos derivados del cruce simple entre el nivel socioeconómico y la posición política ${ }^{5}$. En la diagonal que va desde Izquierda/Bajo a Derecha/

\footnotetext{
${ }^{4}$ Se evaluó la interacción estándar entre orientación política y nivel socioeconómico por medio de análisis de la varianza con mediciones repetidas para incluir las tres mediciones en el tiempo de cada variable dependiente. Solamente con respecto a autoritarismo ( $\mathrm{F}=$ $2,712)$ y apoyo a la democracia $(\mathrm{F}=2,957)$ se obtienen resultados estadísticamente significativos $(\mathrm{p}<0,05)$. Estas dos interacciones, sin embargo, son débiles, y en su conjunto los análisis no ofrecen evidencia de una interacción sistemáticamente entre estos factores. Estos análisis, en cambio, permiten sostener que hay sistemáticamente dos efectos principales independientes, uno de la orientación política y otro del nivel socioeconómico.

${ }^{5}$ En esta sección los datos utilizados corresponden a la segunda medición, que presenta una situación intermedia de lo que sucede en las mediciones 1 y 3 , tanto respecto al $\mathrm{N}$ como respecto al comportamiento en las medidas en que hay diferencias en el tiempo.
} 
Alto se encuentran los llamados grupos congruentes, mientras que los que se separan de esta diagonal son los llamados incongruentes. Respecto de la cantidad de sujetos por celda, puede apreciarse que en todos los niveles socioeconómicos el grupo predominante es el centro. Después del centro, en el nivel alto el $\mathrm{N}$ más alto lo tiene la derecha y en el bajo la izquierda (aunque no es una gran diferencia). Vale decir, la mayoría de los sujetos se congregan en los grupos congruentes.

\section{Tabla 6: Número de sujetos en cruce de orientación política} y nivel socioeconómico

\begin{tabular}{|l|c|c|c|}
\hline & Izquierda & Centro & Derecha \\
\hline Bajo & 49 & 172 & 42 \\
\hline Medio & 56 & 213 & 52 \\
\hline Alto & 19 & 94 & 64 \\
\hline
\end{tabular}

En la Tabla 7 se muestran los promedios de cada uno de los nueve grupos en las actitudes políticas. Para mayor claridad, hemos presentados los promedios para cada actitud política en puntaje estandarizado (z), de tal modo que las desviaciones respecto del valor cero pueden interpretarse como valores superiores (positivos) o inferiores (negativos) al promedio muestral. Puede notarse que, en la mayoría de las variables los grupos congruentes tienen un promedio cercano a 0 , vale decir, parecido al valor predominante en la cultura política chilena.

Tabla 7: Promedio en puntaje $\mathrm{z}$ en actitudes políticas para cada cruce de orientación política con nivel socioeconómico

\begin{tabular}{|l|c|c|c|c|c|}
\hline & Autoritarismo & $\begin{array}{c}\text { Apoyo a la } \\
\text { democracia }\end{array}$ & $\begin{array}{c}\text { Tradiciona- } \\
\text { lismo }\end{array}$ & Nacionalismo & $\begin{array}{c}\text { Temor al } \\
\text { desorden }\end{array}$ \\
\hline Bajo/izq & $\mathbf{- 0 , 0 9 3}$ & $\mathbf{0 , 5 0 7}$ & $\mathbf{- 0 , 0 8 8}$ & $\mathbf{- 0 , 0 1 8}$ & $\mathbf{- 0 , 1 7 2}$ \\
\hline bajo/centro & 0,359 & $-0,294$ & 0,374 & 0,294 & 0,362 \\
\hline bajo/der & 0,684 & $-0,650$ & 0,677 & 0,437 & 0,506 \\
\hline medio/izq & $-0,733$ & 0,685 & $-0,752$ & $-0,472$ & $-0,547$ \\
\hline medio/centro & $\mathbf{- 0 , 0 5 4}$ & $\mathbf{0 , 0 0 1}$ & $\mathbf{- 0 , 0 9 4}$ & $\mathbf{- 0 , 0 3 9}$ & $\mathbf{- 0 , 0 3 8}$ \\
\hline medio/der & 0,188 & $-0,502$ & 0,220 & 0,205 & 0,189 \\
\hline alto/izq & $-1,187$ & 1,150 & $-1,202$ & $-0,669$ & $-1,120$ \\
\hline alto/centro & $-0,643$ & 0,531 & $-0,424$ & $-0,364$ & $-0,419$ \\
\hline alto/der & $\mathbf{0 , 1 6 1}$ & $\mathbf{- 0 , 1 4 1}$ & $\mathbf{0 , 1 5 9}$ & $\mathbf{0 , 0 6 3}$ & $\mathbf{0 , 0 8 6}$ \\
\hline
\end{tabular}

Nota: en negrita se presentan los resultados de los grupos congruentes. 
Con la finalidad de probar esta afirmación hemos construido un índice de congruencia para dar cuenta en detalle de la acción conjunta que tendrían el nivel socioeconómico y la orientación política. Este índice de congruencia se construyó con la resta de los puntajes en ambas variables (con la orientación política reducida a tres valores), de modo que a cada grupo se le asignó un valor (nivel socioeconómico menos orientación política) tal como señala la Tabla 8. Los grupos congruentes tienen un valor de $0 \mathrm{y}$ forman una diagonal que atraviesa los tres niveles socioeconómicos y las tres orientaciones políticas. Los números negativos aparecen en la medida en que la incongruencia se da hacia la derecha en nivel socioeconómico bajo, mientras que los positivos en la medida en que la incongruencia se da hacia la izquierda en nivel socioeconómico alto. Puede advertirse que en este índice mientras más alta es la incongruencia, el valor del índice es más distante a 0 .

\section{Tabla 8: Valores de congruencia asignados a cada cruce de orientación política y nivel socioeconómico}

\begin{tabular}{|l|c|c|c|}
\hline & Izquierda & Centro & Derecha \\
\hline Bajo & 0 & -1 & -2 \\
\hline Medio & 1 & 0 & -1 \\
\hline Alto & 2 & 1 & 0 \\
\hline
\end{tabular}

Hipotetizamos que existirán diferencias respecto a la cultura política chilena (que tiene un promedio de 0 en cada una de las variables estandarizadas) en aquellos grupos que presenten mayor grado de incongruencia entre su nivel socioeconómico y su posición política, mientras que los grupos congruentes tendrían niveles en las actitudes políticas que no se distinguirían de los de la cultura política chilena en general. En otras palabras, los niveles en las actitudes serán semejantes al promedio poblacional en los grupos congruentes y diferentes al promedio poblacional en los grupos incongruentes. En la Tabla 9se muestran los resultados de las pruebas $t$ de muestras independientes realizadas para todos los niveles del índice de congruencia en cada una de las actitudes políticas. Se compara el promedio de los grupos que presentan determinado valor de congruencia con el promedio poblacional (0) que caracteriza la cultura política chilena. Según se observa, los grupos altamente incongruentes y parcialmente incongruentes (por ambos lados, positivo y negativo, con valores 2, 1, - 1 y -2) presentan diferencias estadísticamente significativas con el promedio poblacional en todas las variables. Por el contrario, los grupos congruentes no presentan diferencias significativas con el promedio poblacional. Al hacer el análisis desagregando los grupos congruentes esta situación persiste, vale decir, sin importar que entre los grupos congruentes hay uno de izquierda, uno de centro y otro de 
derecha, no hay diferencias estadísticamente significativas entre estos grupos (juntos o por separados) y el promedio de la muestra. En otras palabras, los grupos congruentes no se diferencian ideológicamente del grueso de la población, están alineados con la cultura política predominante.

Por el contrario, aquellos grupos incongruentes son los que presentan los valores extremos en la mayoría de las actitudes políticas. Los grupos de nivel socioeconómico bajo y de derecha son los que presentan mayores índices de “derechismo” en sus actitudes políticas, mientras que los de nivel socioeconómico alto de izquierda son los que presentan mayores índices de "izquierdismo".

\section{Tabla 9: Pruebas t de muestras independientes para cada valor de congruencia y el promedio de la muestra (valor 0, pues se usan los puntajes z)}

\begin{tabular}{|c|c|c|c|c|c|c|c|c|c|c|c|c|c|c|}
\hline $\begin{array}{l}\text { Interacción } \\
\text { congruente }\end{array}$ & \multicolumn{3}{|c|}{-2} & \multicolumn{3}{|c|}{-1} & \multicolumn{2}{|c|}{0} & \multicolumn{3}{|c|}{1} & \multicolumn{3}{|c|}{2} \\
\hline & \multicolumn{3}{|c|}{$\mathrm{t} \quad \mathrm{gl}$} & \multicolumn{3}{|c|}{$\mathrm{t} \quad \mathrm{gl}$} & \multicolumn{2}{|c|}{$\mathrm{t} \quad \mathrm{gl}$} & \multicolumn{3}{|c|}{$\mathrm{t} \quad \mathrm{gl}$} & \multicolumn{3}{|c|}{$\begin{array}{ll}\mathrm{t} & \mathrm{gl}\end{array}$} \\
\hline Autoritarismo & 5,02 & $* * *$ & 41 & 5,79 & $* * *$ & 222 & $-0,38$ & 323 & $-7,31$ & $* * *$ & 149 & $-4,51$ & $* * *$ & 18 \\
\hline $\begin{array}{l}\text { Apoyo a la } \\
\text { democracia }\end{array}$ & $-4,73$ & $* * *$ & 41 & $-5,76$ & $* * *$ & 221 & 0,97 & 324 & 8,20 & *** & 148 & 7,74 & *** & 18 \\
\hline Tradicionalismo & 5,12 & $* * *$ & 41 & 5,40 & $* * *$ & 222 & $-0,90$ & 323 & $-7,07$ & $* * *$ & 149 & $-6,90$ & $* * *$ & 18 \\
\hline Nacionalismo & 3,95 & $* * *$ & 41 & 4,58 & $* * *$ & 221 & $-0,29$ & 323 & $-4,84$ & $* * *$ & 149 & $-2,21$ & * & 18 \\
\hline Temor al desorden & 4,33 & $* * *$ & 41 & 5,68 & $* * *$ & 221 & $-0,67$ & 323 & $-5,26$ & $* * *$ & 149 & $-4,87$ & $* * *$ & 18 \\
\hline
\end{tabular}

*** $\mathrm{p}<0,001 ;{ }^{* *} \mathrm{p}<0,01 ;{ }^{*} \mathrm{p}<0,05$

Para poner a prueba la forma de la interacción que hemos descrito, basada en el índice aritmético de nivel socioeconómico menos orientación política, construimos una serie de regresiones en las que predecimos las actitudes políticas a partir de los ingresos, la orientación política (en un primer paso) y el índice de congruencia (en el segundo paso). Vale decir, evaluamos en qué medida el índice de congruencia permite explicar las variaciones en las actitudes políticas, comparándolo con los efectos de los ingresos y la orientación política por separadas. La

La Tabla 10muestra los coeficientes de estas regresiones. Se observa que para todas las actitudes el modelo gana poder predictivo al incorporar la congruencia, o sea, al incorporar la congruencia se pueden explicar de mejor forma las variaciones en las actitudes (ver F change en Tabla 10), $y$ en todas, excepto en nacionalismo, la congruencia es el mejor predictor, $\mathrm{o}$ sea, la congruencia permite explicar mejor que los otros dos factores por separado las diferencias en los niveles de las actitudes políticas. 
Tabla 10: Coeficientes del modelo de regresión jerárquica que incorpora la interacción congruente en el segundo paso

\begin{tabular}{|l|c|r|r|r|r|r|r|r|r|}
\hline & $\mathrm{R}^{2}$ ajustado & F change & \multicolumn{5}{c|}{$\beta$ estandarizado } \\
\hline & & & & \multicolumn{3}{|c|}{ Ingresos } & \multicolumn{2}{|c|}{$\begin{array}{l}\text { Orientación } \\
\text { Política }\end{array}$} & \multicolumn{2}{l|}{ Congruencia } \\
\hline Autoritarismo & 0,192 & 25,326 & $* * *$ & $-0,116$ & $*$ & 0,142 & $* *$ & $-0,300$ & $* * *$ \\
\hline Apoyo a la democracia & 0,451 & 26,525 & $* * *$ & 0,064 & & $-0,182$ & $* * *$ & 0,307 & $* * *$ \\
\hline Tradicionalismo & 0,158 & 24,303 & $* * *$ & $-0,063$ & & 0,123 & $*$ & $-0,300$ & $* * *$ \\
\hline Nacionalismo & 0,098 & 4,158 & $*$ & $-0,142$ & $*$ & 0,179 & $* *$ & $-0,129$ & $* *$ \\
\hline Temor al desorden & 0,131 & 14,783 & $* * *$ & $-0,110$ & $*$ & 0,124 & $*$ & $-0,238$ & $* * *$ \\
\hline
\end{tabular}

${ }^{* * *} \mathrm{p}<0,001 ; * * \mathrm{p}<0,01 ;{ }^{*} \mathrm{p}<0,05$

En conclusión, la posición en la estructura social por sí sola no es suficiente para explicar el posicionamiento ideológico de las personas. La inconsistencia entre las actitudes y la orientación política pone en evidencia esta situación. La propuesta de un modelo de interacción entre el nivel socioeconómico y la orientación política basado en la congruencia entre ambas presenta evidencia favorable, mostrándose como un mejor predictor de la configuración ideológica que los ingresos o la orientación política por separado. Para ilustrar que dicho modelo permite dar cuenta de los datos, en la Figura 2 se grafican los promedios grupales de autoritarismo como función de los niveles de congruencia, obteniéndose un decremento homogéneo de esta actitud desde el extremo de incongruencia negativa (nivel socioeconómico bajo y orientación de derecha), pasando por grupos más congruentes, hasta el extremo de incongruencia positiva (nivel socioeconómico alto y orientación de izquierda). Las gráficas correspondientes a los promedios de tradicionalismo, temor al desorden y nacionalismo son muy similares, por lo cual se ha optado por no incluirlas en la Figura 2. Inversamente, y tal como se esperaba, la gráfica de los promedios de apoyo al sistema muestra un incremento homogéneo de esta actitud a medida que pasamos de los valores más negativos a los más positivos del factor de congruencia. En síntesis, la combinación de orientación política y nivel socioeconómico conforme a este modelo de congruencia permite identificar un orden lineal simple en la configuración de actitudes políticas, a condición de complejizar la relación entre dichos factores. 


\section{Figura 2: Promedios en actitudes políticas según niveles de congruencia}

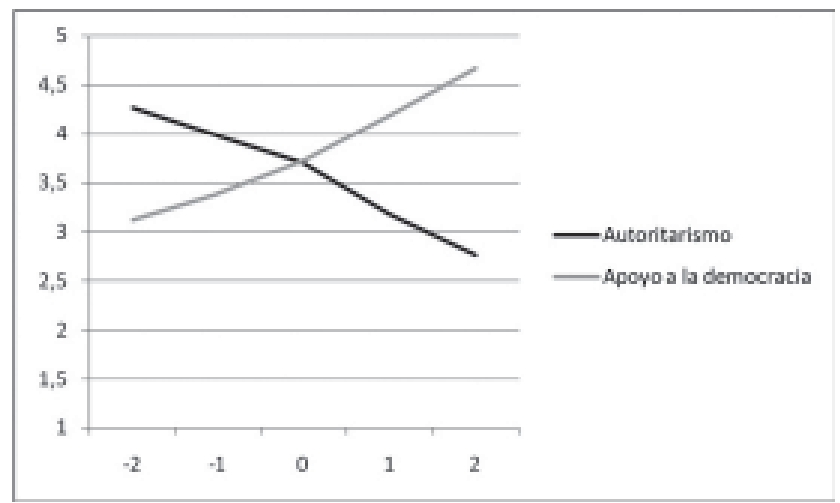

Nota: el patrón en tradicionalismo, temor al desorden y nacionalismo es equivalente al encontrado en autoritarismo.

\section{Discusión}

El estudio permite sostener que la gran mayoría de los chilenos del Gran Santiago comportan un patrón de actitudes políticas compartido, tal como fue descrito. Los niveles de autoritarismo, temor al desorden, etc., son muy similares en grupos distintos, como la derecha de estrato alto, el centro de estrato medio y la izquierda de estrato bajo. La mayor parte de la población cae en dichas categorías, que hemos caracterizado por la congruencia entre su posición ideológica y su posición económica. Esta configuración actitudinal masivamente compartida a través de la sociedad constituye una descripción, parcial pero teóricamente crucial, de la cultura política predominante en nuestro medio. Surgen de inmediato dos preguntas: ¿Cómo llegan a ser tan parecidos en sus actitudes políticas grupos tan dispares? ¿Qué ocurre con los así llamados grupos incongruentes que, si bien representan una minoría de la población, hemos mostrado que comportan importantes desviaciones con respecto a la cultura política predominante?

Los análisis realizados para contestar estas preguntas han abordado sistemáticamente las relaciones entre tres dominios: la orientación política (posicionamiento en el eje izquierda-derecha), la condición socioeconómica, y las actitudes políticas (autoritarismo, apoyo a la democracia, etc.). Hemos mostrado que la relación entre la orientación política y el nivel socioeconómico de las personas, se caracteriza por el aumento del "derechismo" en la medida en que el nivel socioeconómico es más alto, tal como se ha reportado en la literatura (Carney y otros 2008, Napier y Jost 2008). Además, la relación entre la orientación política y el conjunto de actitudes políticas incluidas en el estudio también ha mostrado el patrón esperado de 
acuerdo a la literatura. Vale decir, en las personas de derecha es mayor el autoritarismo (Adorno et al. 1951, Meloen 1993, Stenner 2005), el conservadurismo (en sus dimensiones de temor al desorden y tradicionalismo) (Jost et al. 2003a), y el nacionalismo (Adorno et al. 1950, Billig 1995, Billig y Núñez 1998, Skitk 2005), mientras que el apoyo a la democracia es mayor en las personas de izquierda (Tironi y Agüero 1999, Mainwaring y Torcal 2003, Luna 2008). Por último, la relación entre las actitudes políticas y el nivel socioeconómico mostró resultados concordantes con las premisas de Adorno et al. (1950), Napier y Jost (2008), Schuman et al. (1992), Lipset (1960) y Lipset y Raab (1978), es decir, el autoritarismo y las actitudes asociadas a éste son mayores en los grupos de menores ingresos.

En su conjunto, estos resultados ponen en evidencia una paradoja que no ha sido afrontada por la literatura. Esta paradoja indica, como se ve en la Figura 3, que en los niveles socioeconómicos altos hay mayores índices de actitudes asociadas a la izquierda, pero hay mayor identificación con la derecha, mientras que en el nivel socioeconómico bajo sucede lo contrario.

\section{Figura 3: Relación paradojal entre orientación política, nivel socioeconómico y actitudes políticas.}

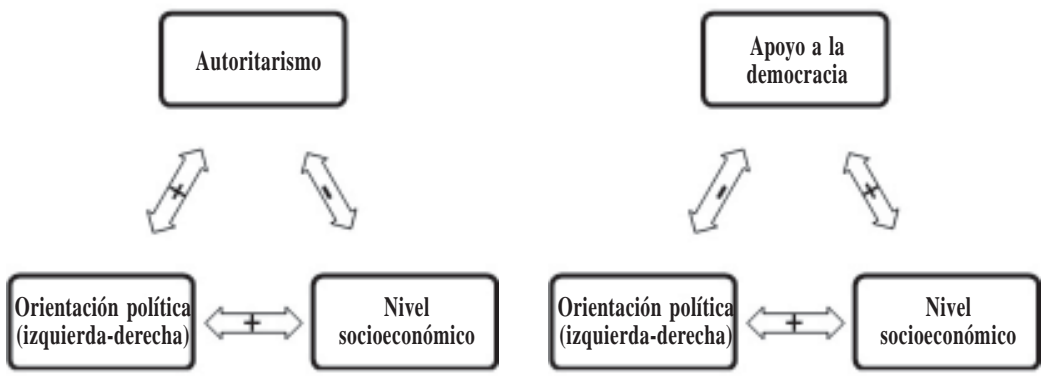

Nota: la relación con autoritarismo es equivalente a la encontrada con tradicionalismo, temor al desorden y nacionalismo.

El argumento central del presente artículo consiste en ofrecer un modelo que resuelve esta paradoja y la evidencia empírica que lo sustenta. Tal como se explicó, las inconsistencias en los resultados se disuelven si es que la orientación política y la condición socioeconómica son consideradas conjuntamente, combinando sus posibles valores y reordenándose en función del grado de congruencia entre ellas. Así, la derecha de estrato alto, la izquierda de estrato bajo y el centro de estrato medio serían los grupos que presentan una orientación política congruente con los intereses de su grupo socioeconómico. Contrariamente, entre la derecha de estrato bajo y 
la izquierda de estrato alto describimos un grado máximo de incongruencia entre la orientación política y los intereses de su grupo económico, aunque esta incongruencia tiene un sentido opuesto. Al reordenar las combinaciones de niveles de orientación política y de acceso económico por medio de esta idea de congruencia, logramos mostrar que los grupos se diferencian, del promedio general de la población en las actitudes políticas, directamente en la medida de su incongruencia y en la dirección en que describe dicha incongruencia. En consecuencia, lo que permite dar cuenta del patrón de actitudes políticas de un grupo no es su orientación política per se, ni menos su extracción social per se, sino el grado de ajuste del posicionamiento ideológico de las personas a sus condiciones socioeconómicas.

\section{Interpretación acerca de la dinámica de la ideología}

Al tener los grupos congruentes los mismos niveles en las actitudes políticas, en cada nivel socioeconómico hay un grupo que adopta el patrón predominante de la cultura política. A su vez, en las clases alta y baja aparecen los grupos que adoptan los niveles extremos en estas actitudes. Conviven entonces en la clase alta un grupo de derecha que presenta el patrón predominante y un grupo de izquierda que muestra los niveles extremos en el sentido pro-democrático, y en la clase baja un grupo de izquierda que presenta el patrón predominante y un grupo de derecha que muestra los niveles extremos en el sentido pro-autoritario o conservador. Sugerimos interpretar este fenómeno como un estado de diferenciación ideológica que opera dentro de las clases: vale decir, los grupos que se diferencian de la cultura política predominante lo hacen respecto de los grupos congruentes de su propia clase. En clase alta, la diferenciación ocurre hacia la izquierda, en referencia al grupo congruente, la derecha; mientras que en clase baja, la diferenciación ocurre hacia la derecha, en referencia al grupo congruente, la izquierda.

Lo anterior permite sostener que la diferenciación en el eje izquierda-derecha (en el sentido de Jost 2006), pierde su carácter paradojal (de acuerdo a lo expuesto en la Figura 3) si asume que opera al interior del grupo socioeconómico, por referencia al grupo congruente. Y, por lo mismo, debe entenderse a las identidades políticas izquierda y derecha en el contexto de un grupo socioeconómico; por ejemplo, no es lo mismo ser de izquierda en el nivel bajo que en el alto, pues en el primer caso se adhiere al patrón predominante y en el segundo se diferencia.

De esta manera, debemos entender la congruencia como el ajuste de la orientación política a las condiciones económicas. La orientación política implica una toma de posición ideológica frente a los valores, creencias y actitudes predominantes en el grupo de referencia, o sea grupo socieconómico. La toma de posición puede ser básicamente de adhesión a la cultura política predominante o de distanciamiento de ésta. Lo ideológico, entendido de esta forma, surge específicamente como la diferenciación interna de la cultura política. 
El fenómeno de las posiciones de derecha exacerbadas en la clase baja ha sido objeto de atención de la literatura, particularmente por la teoría de la justificación del sistema (Jost y Hunyady 2002, 2005, Jost et al. 2004) y por algunos teóricos del autoritarismo (Lipset 1960, Lipset y Raab 1978, Napier y Jost 2008, Schuman et al. 1992). Sin embargo, estas teorías no dan cuenta del fenómeno opuesto: el posicionamiento de la izquierda de clase alta, que presenta los niveles más bajos en las mismas variables en que la derecha de clase baja presenta los niveles más altos. En nuestra interpretación quedan explicados los dos lados del fenómeno.

\section{Falsa conciencia y anclaje psicológico de la ideología}

Los resultados nos llevan a especular que el posicionamiento es una respuesta que los sujetos realizan en función del lugar que ocupan en la organización de la sociedad. Dicho de otro modo, el concepto de ideología que proponemos debe entenderse como resultado de la inseparable relación entre el lugar ocupado en la jerarquía social y la tendencia de quienes ocupan ese lugar a justificar o rechazar la relación jerárquica. Por esta razón, proponemos en primer lugar que es necesario abordar este fenómeno dando cuenta de las relaciones de dominación/subordinación entre los grupos y las tomas de posición ideológicas frente a dicha relación, rescatando así el concepto de clase utilizado tempranamente en la discusión sobre ideología. En segundo lugar, nuestros resultados avalan entender el concepto de falsa conciencia, no como una aflicción psicológica y cognitiva como proponen Jost y Banaji (1994) o Jost et al. (2008), sino como un fenómeno fundado en la realidad social misma, en particular en la estructura de las clases sociales (Augoustinos 1999). Este concepto describe el hecho que la gran mayoría de los chilenos comparte de manera muy homogénea una cultura política sin importar diferencias de orientación política o de condición socioeconómica.

Por lo mismo, a pesar de que la izquierda de clase baja y la derecha de clase alta compartan los mismos niveles en las actitudes, la función que cumple su posición ideológica se funda en su lugar en la jerarquía social, que es muy distinto: los mismos niveles actitudinales les permiten por un lado diferenciarse al interior de su respectiva clase y, por otro, mantener el statu quo, ya sea porque les resulta favorable, en el caso de la clase alta, o porque necesitan justificar su propia posición, en el caso de la clase baja (ver Jost et al. 2004). Así, a pesar que se trata del mismo mecanismo de diferenciación ideológica, es esperable que los contenidos o significaciones de las actitudes políticas sean distintos para estos grupos.

Este fenómeno necesita ser estudiado en mayor profundidad, pues la mera observación cuantitativa de las actitudes políticas no da cuenta de la articulación de ideas subyacente a la posición ideológica. A pesar de que el estudio de las actitudes ha mostrado ser un modo fructífero de aproximarse a los aspectos psicológicos de la ideología, llegando incluso a dar cuenta de sus correlatos fisiológicos (por ejemplo Amodio et al. 2007), el 
anclaje psicológico de la ideología no agota todos los aspectos importantes de la ideología, en particular los anclados en las relaciones sociales y en las dinámicas de discurso (Billig et al. 1988). Por lo mismo, sugerimos complementar el enfoque presentado en este artículo con estudios enfocados en la construcción del discurso ideológico, particularmente en grupos extremos (Billig 2002, Billig et al. 1988; por ejemplo Billig 1978).

Proponemos también, que el estudio de la ideología debe considerar una aproximación multinivel, tal como han propuesto Pettigrew (1998, 2006, 2008) Stellmacher y Petzel (2005) y Wagner et al. (2008), que incorpore muestras que representen todos los estratos socioeconómicos, como lo sugiere la evidencia en torno a la importancia de la clase para entender el posicionamiento ideológico. Por lo mismo, los resultados obtenidos en estudios conducidos con muestras universitarias deben ser observados cuidadosamente. Por último, debemos rescatar la importancia del uso de diseños longitudinales que permiten incorporar las dinámicas temporales de las relaciones intergrupales, como sugieren varios autores (Pettigrew y Tropp 2006, van Laar et al. 2008, Pettigrew 2008). 


\section{Bibliografía}

Adorno, Theodor W., Else Frenkel-Brunswik, Daniel Levinson, y Nevitt Sanford (1950), The Authoritarian Personality, Harper and Row, New York.

Adorno, Theodor W., y M. Horkheimer (1969), La sociedad. Lecciones de sociología, Proteo, Buenos Aires.

Almond, G. y Sidney Verba (1965), The civic culture, Princeton University Press, Boston.

Althusser, Louis (1997), “Ideología y aparatos ideológicos del estado”, en La filosofía como arma para la revolución, de Louis Althusser, Siglo XXI, México.

Altmeyer, Robert A (1981), Right-wing authoritarianism, University of Manitoba Press, Winnipeg, Manitoba.

Idem (1998), “The other 'authoritarian personality' ”, en Advances in Experimental Social Psychology 30, 47-91.

Alwin, D.F. y J.A. Krosnick (1991), “Aging, cohorts, and the stability of sociopolitical orientations over the life span”, en American Journal of Sociology 97, 169-195.

Amodio, David M., John T. Jost, Sarah L. Master, y Cindy M. Yee (2007), "Neurocognitive correlates of liberalism and conservatism", en Nature Neuroscience 10(10), 1246-1247.

Augoustinos, Martha (1999), “Ideology, False Consciousness and Psychology”, en Theory y Psychology 9(3), 295-312.

Billig, Michael (1978), Fascists: A social psychological view of the National Front, Academic Press, London.

Idem (1984), "Political ideology: social psychological aspects”, en The social dimension, Vol. 2 (pp. 446-470), Henri Tajfel (Ed.), Cambridge University Press, Cambridge.

Idem (1986), “Racismo, prejuicio y discriminación”, en En Psicología Social, II (pp. 575-600), Serge Moscovici (Ed.), Paidós, Barcelona.

Idem (1995), Banal Nationalism. SAGE Publications, London.

Idem (2002), “Henri Tajfel's 'Cognitive aspects of prejudice' and the psychology of bigotry”, en British Journal of Social Psychology 41, 171-188.

Billig, M., Condor, S., Edwards, D., Gane, M., Middleton, D., y Radley, A (1988), Ideological Dilemmas, Sage Publications, London. 
Billig, Michael y Rosamaría Núñez (1998), “El nacionalismo banal y la reproducción de la identidad nacional”, en Revista Mexicana de Sociología 60(1), 37-57.

Brunner, J.J (1990), “Chile: entre la cultura autoritaria y la cultura democrática”, en Cultura y Política en América Latina (85-98), H. Zemelman (Ed.), Siglo XXI, México.

Carlin, Ryan (2006), “The decline of citizen participation in electoral politics in post-authoritarian Chile”, en Democratization 13(4), 632-651.

Carney, Dana R., John T. Jost, Samuel D. Gosling, y Jeff Potter (2008), "The Secret Lives of Liberals and Conservatives: Personality Profiles, Interactions Styles, and the Things They Leave Behind”, en Political Psychology 29(6), 807-840.

Citrin, J. y Ch. Muste (1993), “Trust in government”, en Measures of political attitudes (pp. 465-531), J.P. Robinson, P.R. Shaver, y L.S. Wrightsman (Eds.), Academic Press, San Diego.

Colomer, Josep, y Luis Escatel (2005), “La dimensión izquierda y derecha en América Latina”, en Desarrollo Económico 45(177), 123-136.

Comrey, Andrew L., y James A. Newmeyer (1965), “Measurement of Radicalism-Conservatism”, en Journal of Social Psychology 67, 357-369.

Dawson, R. y K. Prewitt (1969), Political socialization, Little, Brown \& Company, Boston.

Duncan, Lauren E. y Gail S. Agronick (1995), “The Intersection of Life Stage and Social Events: Personality and Life Outcomes”, en Journal of Personality and Social Psychology 69 (3), 558-568.

Eagly, A. y S. Chaiken (1993), The psychology of attitudes, Harcourt Brace and Jovanovich, Fort Worth.

Feldman, Stanley (2003a), "Enforcing social conformity: A theory of authoritarianism”, en Political Psychology 24, 41-74.

Idem (2003b), “Values, Ideology, and the Structure of Political Attitudes”, en The Oxford Handbook of Political Psychology (pp. 477-508), David O. Sears, Leonie Huddy y Robert Jervis (Eds.), Oxford University Press, New York.

Funke, Friedrich. 2005. “The Dimensionality of Right-Wing Authoritarianism: Lesson from the Dilemma between Theory and Measurement”, en Political Psychology 26(2), 195-218.

Glenn, N. D (1974), “Aging and conservatism”, en Annals of the American Academy of Political and Social Science 415, 176-186. 
Higgins, E. T (1998), "Promotion and prevention: Regulatory focus as a motivational principle”, en Advances in Experimental Social Psychology 30, 1-45.

Jennings, M. y R. Niemi (1981), Generation and politics. A panel study of young adults and their parents, Princeton University Press, Princeton. Jost, John T (2006), "The end of the end of ideology", en American Psychologist 6(7), 651-670.

Jost, John T., Mahzarin R. Banaji, y Brian A. Nosek (2004), “A decade of system justification theory: Accumulated evidence of conscious and unconscious bolstering of the status quo”, en Political Psychology 25(6), 881-919.

Jost, John T., Christopher M. Federico, y Jaime L. Napier (2009), “Political Ideology: Its Structure, Functions, and Elective Affinities”, en Annual Review of Psychology 60, 307-337.

Jost, John T., J. Glaser, A. W. Kruglanski, y F. Sulloway (2003a), “Political conservatism as motivated social cognition”, en Psychological Bulletin 129, 339-375.

Idem (2003b), "Exceptions that prove the rule: Using a theory of motivated social cognition to account for ideological incongruities and political anomalies”, en Psychological Bulletin 129, 383-393.

Jost, John T., y Orsolya Hunyady (2002), “The psychology of system justification and the palliative function of ideology”, en European Review of Social Psychology 13, 111-153.

Idem (2005), “Antecedents and consequences of system-justifying ideologies”, en Current Directions in Psychological Science 14, 260-265.

Jost, John T., Brian A. Nosek, y Samuel D. Gosling (2008), “Ideology. Its Resurgence in Social, Personality, and Political Psychology”, en Perspectives on Psychological Science 3(2), 126-136.

Kemmelmeier, Markus (2008), “Is there a relationship between political orientation and cognitive ability? A test of three hypotheses in two studies", en Personality and Individual Differences 45, 767-772.

Kerlinger, F. N (1984), Liberalism and conservatism: The nature and structure of social attitudes, Erlbaum, Hillsdale.

Knight, Kathleen (1999), “Liberalism and Conservatism”, en En Measures of Political Attitudes (pp. 59-158), John P. Robinson, Phillip R. Shaver y Lawrence S. Wrightsman (Eds), Academic Press, San Diego.

Idem (2006), “Transformations of the concept of ideology in the twentieth century”, en American Political Science Review 100, 619-626. 
Larraín, Jorge (2007), El Concepto de Ideología. Vol. 1: Carlos Marx, LOM, Santiago.

Idem (2008), El Concepto de Ideología. Vol. 2: El marxismo posterior a Marx: Gramsci y Althusser, LOM, Santiago.

Lenk, Kurt (1974), El Concepto de Ideología, Amorrortu, Buenos Aires.

Levin, Shana, James Sidanius, Joshua L. Rabinowitz, y Christopher Federico (1998), "Ethnic identity, legitimizing ideologies, and social status: a matter of ideological asymmetry”, en Political Psychology 19(2), 373-404. Lipset, S. (1960), Political man, Doubleday, New York.

Lipset, S., y E. Raab (1978), The politics of unreason: Right-wing extremism in America, 1790-1977, University of Chicago Press, Chicago.

Lukács, Georg (1969), Historia y conciencia de clase, Grijalbo, México.

Luna, Juan Pablo (2008), “Partidos políticos y sociedad en Chile. Trayectoria histórica y mutaciones recientes”, en Reforma a los partidos políticos en Chile (75-125), Arturo Fontaine y et al. (Eds.), PNUD, Santiago.

Mainwaring, Scott, y Mariano Torcal (2003), “The Political Re-crafting of Social Bases of Party Competition: The Case of Chile 1973-1995”, en British Journal of Political Science 33, 55-84.

Marx, Karl, y Frederick Engels (1845/1974), La ideología alemana, Cultura Popular, México.

Mcdevitt, Michael, y Steven Chaffee (2002), “From Top-Down to TrickleUp Influence: Revisiting Assumptions About the Family in Political Socialization”, en Political Communication, 19, 281-301.

Meloen, J (1993), “The F-scale as a predictor of fascism: an overview of 40 years of authoritarianism research", en Strength and weakness: the authoritarian personality today, W. F. Stone, G. Lederer y R. Christie (Eds.), Springer-Verlag, New York.

Michaud, Kristy E. H., Juliet E. Carlisle, y Eric R. A. N. Smith (2009), “The Relationship between Cultural Values and Political Ideology, and the Role of Political Knowledge”, en Political Psychology 30(1), 27-42.

Ministerio de Planificación, Gobierno de Chile (2006), Encuesta de Caracterización Socioeconómica Nacional, MIDEPLAN, Santiago.

Muller, J. Z (2001), “Conservatism: Historical aspects”, en International encyclopedia of the social and behavioral sciences (pp. 2624-2628), N. J. Smelser y P. B. Baltes (Eds.), Elsevier, Amsterdam. 
Napier, Jaime L., y John T. Jost (2008), “The ‘Antidemocratic Personality” Revisited: A Cross-National Investigation of Working-Class Authoritarianism”, en Journal of Social Issues 64(3), 595-617.

Navia, Patricio (2004), “Participación Electoral en Chile, 1988-2001”, en Revista de Ciencia Política 24(1), 81-103.

Idem (2005), “La transformación de votos en escaños: leyes electorales en Chile, 1833-2004”, en Política y Gobierno 12(2), 233-276.

Idem. 2007. “El pluralismo y el arcoiris de la Concertación”, en Revista UDP Pensamiento y Cultura 3(5), 17-22.

Ortega, Eugenio (2003), “Los partidos políticos chilenos: Cambio y estabilidad en el comportamiento electoral 1990-2000”, en Revista Ciencia Política 22(2), 109-147.

Pettigrew, Thomas F (1998), “Applying social psychology to international social issues”, en Journal of Social Issues 54, 663-675.

Idem (2006), “The advantages of multilevel approaches”, en Journal of Social Issues 62(3), 615-620.

Idem (2008), "Reflections on core themes in intergroup research", en Improving intergroup relations. Building on the legacy of Thomas Pettigrew (pp. 283-303), Ulrich Wagner, Linda R. Tropp, Gillian Finchilescu y Colin Tredoux (Eds.), Blackwell, Oxford.

Pettigrew, Thomas F., y Linda R. Tropp (2006), “A meta-analytic test of intergroup contact theory”, en Journal of Personality and Social Psychology 90, 751-783.

Pratto, Felicia, James Sidanius, y Shana Levin (2006), “Social dominance theory and the dynamics of intergroup relations: Taking stock and looking forward”, en European Review of Socila Psychology 17, 271-320.

Pye, Lucian W. y Sidney Verba (Eds.) (1965), Political culture and political development, Princeton University Press, Princeton.

Robinson, John, Phillip Shaver, y Lawrence Wrightsman (Eds.) (1999), Measures of Political Attitudes, Academic Press, San Diego.

Ross, M (1989), "Relation of implicit theories to the construction of personal histories”, en Psychological Review 96, 341-357.

Schuman, Howard, Lawrence Bobo, y Maria Krysan (1992), "Authoritarianism in the General Population: The Education Interaction Hypothesis”, en Social Psychology Quarterly 55(4), 379-387. 
Schuman, Howard y Jacqueline Scott (1989), "Generations and Collective Memories”, en American Sociological Review 54 (3),359-381.

Sears, David O (1990), "Whither Political Socialization Research? The Question of Persistence”, en Political Socialization, Citizenship Education, and Democracy (pp. 69-97), O. Ichilov (Eds.), Teachers College Press, New York.

Sears, D. y S. Levy (2003), “Childhood and adult political development”, en D. Sears, L. Huddy y R. Jervis (Eds.), Oxford handbook of political psychology, Oxford University Press, Oxford.

Segovia, Carolina, Andrés Haye, Roberto González, Jorge Manzi y Héctor Carvacho (2008), “Confianza en Instituciones Políticas en Chile: Un Modelo de los Componentes Centrales de Juicios de Confianza”, en Revista de Ciencia Política 28(3), 39-60.

Sidanius, James (1993), “The psychology of group conflict and the dynamics of oppression: A social dominance perspective”, en Explorations in political psychology (183-219), S. Iyengar y W. McGuire (Eds.), Duke University Press, Durham.

Sidanius, James, y Felicia Pratto (1999), Social dominance: An intergroup theory of social hierarchy and oppression, Cambridge University Press, New York.

Skitk, Linda J (2005), "Patriotism or Nationalism? Understanding PostSeptember 11, 2001, Flag-Display Behavior”, en Journal of Applied Social Psychology, 35(10), 1995-2011.

Stellmacher, Jost, y Thomas Petzel (2005), “Authoritarianism as a Group Phenomenon”, en Political Psychology 26(2), 245-274.

Stenner, Karen (2005), The Authoritarian Dynamic, Cambridge University Press, New York.

Steward, Abigail J. y Joseph M. Jr. Healy (1989), “Linking Individual Development and Social Changes”, en American Psychologist 44(1), 3042.

Tajfel, H., y J. C. Turner (1986), “The social identity theory of intergroup behavior”, en The psychology of intergroup relations (pp. 7-24), S. Worchel y W. G. Austin (Eds.), Nelson-Hall, Chicago.

Tironi, Eugenio, y Felipe Agüero (1999), “¿Sobrevivirá el nuevo paisaje chileno?” Estudios Públicos 74, 151-168.

Toro, Sergio (2008), “De lo épico a lo cotidiano: Jóvenes y generaciones políticas en Chile”, en Revista de Ciencia Política 28(3), 143-160. 
van Laar, Colette, Shana Levin, y James Sidanius (2008), “Ingroup and Outgroup Contact. A longitudinal study of the effects of cross-ethnic friendships, dates, roommate relationships and participation in segregated organizations”, en Improving intergroup relations. Building on the legacy of Thomas F. Pettigrew (pp. 127-142), Ulrich Wagner, Linda R. Tropp, Gillian Finchilescu y Colin Tredoux (Eds.), Blackwell, Oxford.

Wagner, Ulrich, Oliver Christ, Hinna Wolf, Rolf van Dick, Jost Stellmacher, Elmar Schlüter, y Andreas Zick (2008), "Social and political context effects on intergroup contact and intergroup attitudes", en Improving intergroup relations. Building on the legacy of Thomas F. Pettigrew (pp. 195-209), Ulrich Wagner, Linda R. Tropp, Gillian Finchilescu y Colin Tredoux (Eds.), Blackwell, Oxford.

Wilson G. D (1973), The Psychology of Conservatism, Academic Press, London. 